\title{
Produção de Ocratoxina A por Aspergillus Carbonarius em Meio À Base de Cacau
}

\author{
Luisa Freire (I), Fabiana Reinis Franca Passamani (I), Danieli \\ Cristina Schabo (II), Camila Alvarenga Freire (I), Luis Roberto \\ Batista (I)
}

(I) UFLA - Universidade Federal de Lavras (Câmpus Universitário, Caixa Postal 3037, CEP 37200-000, Lavras/MG), (II) INFRO - Instituto Federal de Rondônia (Rodovia 399, Km 05 Zona Rural Cidade: Colorado do Oeste - RO CEP: 76993-000)

\section{Resumo}

A qualidade dos produtos obtidos do cacau depende de fatores ambientais, agronômicos e tecnológicos. Tais fatores poderão interferir no desenvolvimento de fungos potencialmente toxigênicos e na presença de ocratoxina A (OTA) em amêndoas de cacau. Este estudo teve como objetivo avaliar a influência da temperatura e atividade de água na produção de OTA por Aspergillus carbonarius em meio de cultura à base de amêndoa de cacau. O fungo testado foi isolado de uma amostra de amêndoa de cacau e faz parte do acervo da Coleção de Cultura de Microrganismos do Departamento de Ciência dos Alimentos - UFLA. O isolado foi reativado em meio $\mathrm{MA}$ e incubado a $25^{\circ} \mathrm{C}$ por 7 dias. $\mathrm{A}$ determinação do potencial toxigênico foi feita através do teste do Plug Agar por Cromatografia de Camada Delgada. Após a confirmação do potencial ocratoxigênico, o fungo foi inoculado em meio à base de cacau com diferentes atividades de água $(0,91,0,93,0,96$ e 0,99$)$ e incubado nas temperaturas $10^{\circ} \mathrm{C}, 15^{\circ} \mathrm{C}, 27,5^{\circ} \mathrm{C}, 40^{\circ} \mathrm{C} \mathrm{e} 45^{\circ} \mathrm{C}$. O experimento foi realizado em duplicata. A quantificação de OTA foi realizada por Cromatografia Líquida de Alta Eficiência. Utilizou-se o teste de médias, ao nível de 5\% de significância para avaliar a diferença da produção de OTA entre os ensaios. Foram observadas diferenças no nível de produção de OTA com as alterações nas condições de cultivo. A condição que propiciou uma maior produção de OTA por A. carbonarius foi $15^{\circ} \mathrm{C}$ e 0,99aw $(2,36 \mu \mathrm{g} / \mathrm{g})$. Os

\footnotetext{
Referência:

Luisa Freire, Fabiana Reinis Franca Passamani, Danieli Cristina Schabo, Camila Alvarenga Freire, Luis Roberto Batista. Produção de Ocratoxina A por Aspergillus Carbonarius em Meio À Base de Cacau. In: Anais do 12은 Congresso Latinoamericano de Microbiologia e Higiene de Alimentos - MICROAL 2014 [= Blucher Food Science Proceedings, num.1, vol.1]. São Paulo: Editora Blucher, 2014. 
ensaios $40^{\circ} \mathrm{C}$ e $0,93 \mathrm{aw}(0,00 \mu \mathrm{g} / \mathrm{g}), 40^{\circ} \mathrm{C}$ e $0,99 \mathrm{aw}(0,00 \mu \mathrm{g} / \mathrm{g}), 10^{\circ} \mathrm{C}$ e $0,96 \mathrm{aw}(0,00 \mu \mathrm{g} / \mathrm{g}), 45^{\circ} \mathrm{C}$ e $0,96 \mathrm{aw}(0,00 \mu \mathrm{g} / \mathrm{g}), 15^{\circ} \mathrm{C}$ e $0,93 \mathrm{aw}(0,11 \mu \mathrm{g} / \mathrm{g})$, $27,5^{\circ} \mathrm{C}$ e $0,91 \mathrm{aw}(0,26 \mu \mathrm{g} / \mathrm{g})$ foram os que propiciaram piores condições para produção de OTA. Já nos ensaios $27,5^{\circ} \mathrm{C}$ e $0,99 \mathrm{aw}, 27,5^{\circ} \mathrm{C}$ e $0,96 \mathrm{aw}$ o isolado foi capaz de produzir 1,07 e $1,81 \mu \mathrm{g} / \mathrm{g}$, respectivamente. Estes resultados demonstram que A. carbonarius é capaz de produzir OTA em uma ampla faixa de atividades de água e temperatura. Esses dados sugerem o risco potencial de produção de OTA por A. carbonarius, em amêndoas de cacau devido às condições propiciadas pelas regiões de plantio no Brasil. Além disto, também contribuem para uma melhor compreensão da ecofisiologia desta espécie. Conhecendo as condições ideais de produção de OTA e em quais etapas de processamento estas condições são propicias é possível avaliar o risco potencial para a saúde proporcionado pelo consumo de produtos derivados do cacau.

Palavras-Chave: Cacau, Ocratoxina A, Aspergillus carbonarius Agência de Fomento: Cnpq, Fapemig 